PROFESIONALES Y HERRAMIENTAS PARA EL DESARROLLO LOCAL Y SUS SINERGIAS TERRITORIALES. EVALUACIÓN Y PROPUESTAS DE FUTURO IX Coloquio Nacional de Desarrollo Local del GTDL-AGE 

ANTONIO MARTÍNEZ PUCHE, XAVIER AMAT MONTESINOS, ISABEL SANCHO CARBONELL y DANIEL SANCHIZ CASTAÑO (EDS.)

\section{PROFESIONALES Y HERRAMIENTAS PARA EL DESARROLLO LOCAL Y SUS SINERGIAS TERRITORIALES. EVALUACIÓN Y PROPUESTAS DE FUTURO}

IX Coloquio Nacional de Desarrollo Local del GTDL-AGE

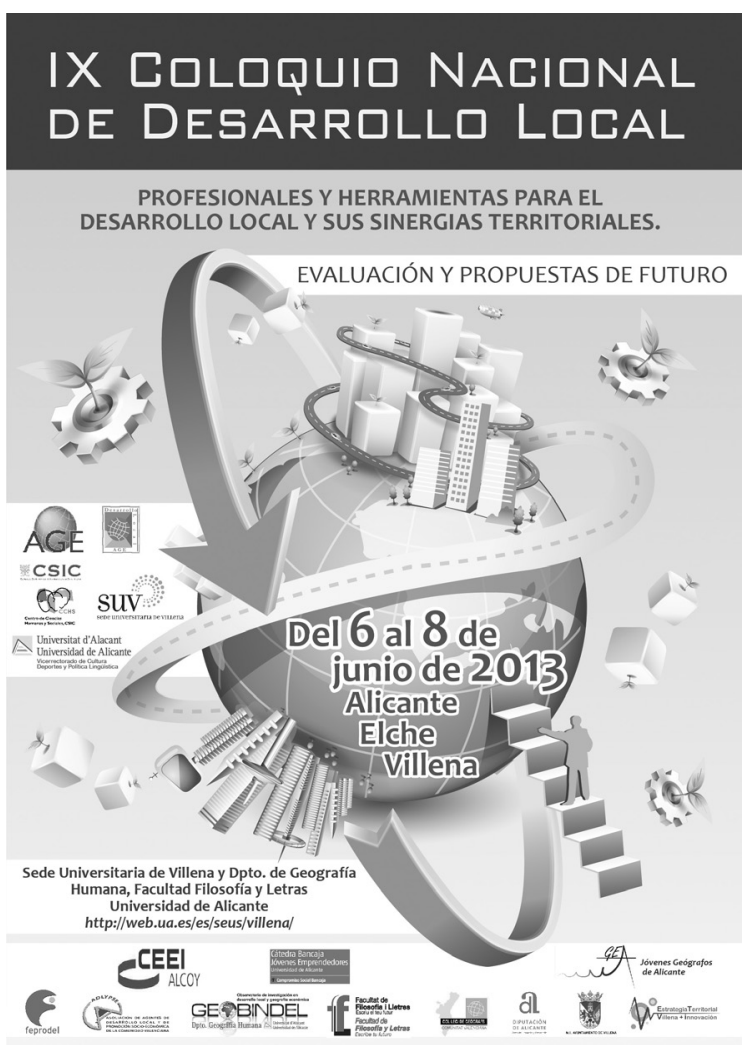


Este libro ha sido examinado y valorado por evaluadores ajenos a la Universidad de Alicante, con el fin de garantizar la calidad científica del mismo.

Publicacions de la Universitat d'Alacant

03690 Sant Vicent del Raspeig

Publicaciones@ua.es

http://publicaciones.ua.es

Telèfon: 965903480

(C) Antonio Martínez Puche, Xavier Amat Montesinos,

Isabel Sancho Carbonell y Daniel Sanchiz Castaño (eds.), 2016

(C) d'aquesta edició: Universitat d'Alacant

ISBN: 978-84-16724-00-0

Dipòsit legal: A 92-2016

Disseny de coberta: candela ink

Composició: Página Maestra (Miguel Ángel Sánchez Hernández)

Impressió i enquadernació: Guada Impresores

\section{unte \\ Unión de Editoriales
Universitarias Españolas \\ WWW.une.es
WWA}

Esta editorial es miembro de la UNE, cosa que garantiza la difusión y comercialización nacional y internacional de sus publicaciones.

Reservados todos los derechos. Cualquier forma de reproducción, distribución, comunicación pública o transformación de esta obra sólo puede ser realizada con la autorización de sus titulares, salvo excepción prevista por la ley. Diríjase a CEDRO (Centro Español de Derechos Repográficos, www.cedro.org) si necesita fotocopias o escanear algún fragmento de esta obra. 


\title{
AGRICULTURA SOCIAL: ECONOMÍA SOCIAL Y DINAMIZACIÓN AGROECOLÓGICA COMO ESTRATEGIA DE DESARROLLO LOCAL. EL CASO DE L'OLIVERA COOPERATIVA (URGELL, CATALUÑA)
}

\author{
Carles Guirado González \\ Departament de Geografia. Universitat Autònoma de Barcelona \\ carlesguirado@gmail.com \\ Anna Badia i Perpinyà \\ Departament de Geografia. Universitat Autònoma de Barcelona \\ anna.badia@uab.cat \\ Antoni Francesc Tulla i Pujol \\ Departament de Geografia. Universitat Autònoma de Barcelona \\ Anna Vera Martín \\ Departament de Geografia. Universitat Autònoma de Barcelona
}

\section{Resumen}

La Agricultura Social (AS) ha surgido como un sector emergente en el mundo rural europeo en los últimos años. El impulso de la AS se caracteriza por la atribución de nuevas funcionalidades y significados a la práctica agrícola, más allá de la propia función productiva. El surgimiento de estas iniciativas agrícolas innovadoras contribuye al desarrollo local, a la formulación de alternativas económicas y muestran la aparición de un nuevo paradigma agrosocial. En esta comunicación realizaremos una breve contextualización del fenómeno y analizaremos el caso de l'Olivera Cooperativa.

Palabras clave: Agricultura social, dinamización agroecológica, desarrollo local, agricultura multifuncional. 


\title{
SOCIAL FARMING: SOCIAL ECONOMY AND AGROECOLOGICAL DYNAMIZATION AS A LOCAL DEVELOPMET ESTRATEGY. L'OLIVERA COOPERATIVA AS STUDY CASE (URGELL, CATALONIA)
}

\begin{abstract}
Social Farming (SF) is an emerging sector of the rural European context. The most important motivating force for this activity has been the new functions and meanings that have quite recently been acquired by agricultura, beyond the traditional prouctive function. As a result, innovative SA activities are contributing to the local development, supponrt for a new agro-social paradugm and show economy alternatives. In this we address the SA phenomena and analyze the Olivera Cooperativa case.
\end{abstract}

Key words: social farming, agroecological dynamization, lcoal development, multifunctional agriculture.

\section{INTRODUCCIÓN}

El impulso que en las últimas dos décadas ha experimenta la Agricultura Social (en adelante AS) en Europa debe relacionar con procesos socioculturales y cambios en las tendencias y pautas de la población, principalmente surgidas en las sociedades postindustriales. Tras el surgimiento y desarrollo de actividades innovadoras, tendencias y pautas económicas emergentes, siempre hay cambios sociales y culturales que los apoyan y les dan sentido. El cambio en la situación en los territorios rurales, junto con la emergencia de un nuevo campesinado, las innovaciones sociales y las nuevas tendencias en el sector de los servicios, han contribuido a tejer nuestras alianzas entre agricultura y sociedad. En este contexto, la AS aparece como una estrategia para ofrecer soluciones y respuesta a problemas y necesidades presentes en la sociedad actual, las cuales surgen en la mayoría de las ocasiones desde la implicación y participación de la ciudadanía, con el fin de subsanar las carencias (y a menudo ineficacia) de las políticas públicas en la esfera de lo social y del desarrollo local.

El espacio agrario lleva experimentando, desde hace algunas décadas profundas transformaciones (Woods, 2005). La industrialización de la agricultura después dela II Guerra Mundial conllevó importantes cambios en las explotaciones agrarias tradicionales, las cuales se centraron en obtener el máximo rendimiento de la producción (Browler, 1992; Lockwood, 1999), relegando aquellas que no eran "competitivas" a un lugar marginal dentro del sector (Ploeg, 2006). A partir de la década de 1980, la transición productivista va perdiendo empuje y se inicia el cambio hacia una nueva etapa llamada postproductivismo, en la que se incorporan a la agricultura nuevas funciones que permite diversificar la economía rural con actividades principalmente relacionadas con 
el sector terciario, dando lugar a lo que se ha denominado agricultura multifuncional, multidimensional o polivalente (Armesto, 2005). Paralelamente, aparece u nuevo paradigma de dinamización agroecológica, en el que surge un nuevo campesinado (Ploeg, 2010), con una conciencia renovada, nuevos valores y nuevas manteras de producir, que ayudan a redefinir el papel de la agricultura en el seno de la sociedad actual. Esta nueva agricultura apuesta por un modelo agroalimentario contestatario que vela por el acceso directo a productos producidos de manera ecológica, con la mínima carca energética posible y con valor social añadido, a los que los consumidores aceden a través de la compra directa en la explotación, grupos y cooperativas de consumo y establecimientos especializados, materializando un consumo responsable como acción consciente de crítica y denuncia al sistema agroalimentario imperante (López, Fernández, 2004; Carbone, Gaito, Senni, 2007).

\section{2. ¿QUÉ ES LA AGRICULTURA SOCIAL?}

La AS es un tema emergente y un sector dinámico entre los diferentes actores de los territorios rurales en Europa (campesinado, organizaciones de apoyo a la agricultura, usuarios, consumidores, Tercer Sector Social y Administración pública). El interés por esta actividad radica en la percepción sobre la potencialidad y el impacto positivo que tiene la AS sobre el desarrollo de la agricultura y el aprovechamiento de los recursos agrarios, no sólo en su vertiente productiva, sino también la esfera de lo social, apareciendo como una potente estrategia de desarrollo local y una apuesta por una economía social, ética y solidaria. En este sentido, la AS aparece como una oportunidad para diversidad las actividades en el sí de las áreas rurales y las propias explotaciones agrarias, ofreciendo nuevos servicios y proporcionando al campesinado nuevas fuentes de ingresos, a la vez que redefinen su papel en el imaginario social y en el marco de las estrategias de desarrollo rural, actuando como proveedores de servicios asistenciales y de integración social (O'Connor, Lai, Watson, 2010).

La AS es un concepto derivado del término inglés Social Farming, aunque frecuentemente recibe otros apelativos (Green care, farming for health, civic agricultura), haciendo difícil abordar su conceptualización y generando, a menudo, una cierta imprecisión terminológica. A grandes rasgos, se puede decir que la AS incluye todas las actividades que emplean los recursos agrarios con el fin de promover (o generar) actividades y/o servicios terapéuticos, de rehabilitación, de inclusión social, educativo y de formación de inserción laboral, principalmente en las áreas rurales y periurbanas (Di Iacovo, O'Connor, 2009).

Después de revisar una extensa bibliografía sobre la AS hemos percibido la inexistencia de una definición o un tratamiento consensuado del concepto, lo que nos ha obligado a establecer una definición que sea operativa para nuestra investigación, adaptando las aportaciones de diferentes autores a nuestro 
contexto geográfico. En este sentido, entendemos la AS como el conjunto de experiencias que emplean los recursos locales, agrarios y/o naturales, para promover la salud, el empleo y empoderamiento de colectivos en riesgo de marginación social y la generación de servicios sociosanitarios en las áreas rurales y periurbanas, a través de la práctica agraria y actividades derivadas. Además, es importante destacar, como la presencia de estas prácticas contribuyen a la innovación y a la multifuncionalidad agraria, a la construcción de modelos económicos alternativos, al diseño de nuevas estrategias de desarrollo local y a la creación de nuevas formas de organización que fomentan la cooperación entre agentes del territorio y la sociedad, con el fin de promover la inserción laboral, la cohesión social y el bienestar físico y mental de las personas.

Las experiencias de AS presentan una serie de características que nos ayudan a delimitar el concepto. La mayoría de las explotaciones agrarias que practican la AS suelen ser de carácter familiar o de dimensiones reducidas, permitiendo desarrollar una organización del trabajo de forma conjunta y cooperativa y fomentar la relación en grupos reducidos, en contacto con la naturaleza, en un ambiente agradable y en armonía con el resto de trabajadores/as de la finca y con los facilitadores/as que apoyan a los colectivos beneficiarios -como por ejemplo, personas con discapacidad, en riesgo de exclusión social, drogodependientes, jóvenes, etc.- para alcanzar los objetivos marcados. Es por ello que la AS no contempla explotaciones enfocadas a una agricultura intensiva e industrial, ya que en estos entornos laborales no es posible encontrar el ambiente ni los elementos adecuados para alcanzar los objetivos propios que esta actividad se plantea. Creemos que la práctica de la AS debe ir más allá del valor social, y debe abarcar también la voluntad de llevar a cabo una agricultura respetuosa con el medio ambiente, de proximidad; que cuide de las personas con las que trabaja y de las que consumirán sus productos, pero también de lo que está produciendo (variedades locales, productos de temporada) y como lo está haciendo (sin pesticidas, siguiendo los ritmos y ciclos propios de la tierra, en extensivo). Es por este motivo que a menudo uno de los elementos que definen las explotaciones de AS es la producción ecológica y el fomento de un consumo responsable y de proximidad (Carbone, Senni, 2010; Medina, 2010).

En el caso específico de las áreas rurales los proyecto de AS son una respuesta clara a la penalización que estos territorios han surgido durante décadas por su emplazamiento periférico y alejado de los grandes núcleos urbanos y a las limitaciones sociales del modelo de desarrollo neoliberal, en los que las posibilidad de desarrollo se han centrado en el ocio y la construcción en los que existe una importante infradotación de servicios, especialmente de carácter social (Collantes, 2005). La situación resultante de estos cambios en las áreas rurales ha hecho crecer la necesidad de desarrollar sistemas de producción agrícola más sostenibles, que respondan a las necesidades de la sociedad y que integren valores sociales, ambientales, territoriales y económicos. Es por ello 
que creemos que la AS es una práctica agrícola innovadora que deja patente la emergencia de un nuevo paradigma agrosocial (Sevilla, 2007) y el surgimiento de un nuevo campesinado (Monllor, 2011), ofreciendo la posibilidad de compaginar práctica agrícola y contribución social, participando no sólo del sistema productivo, sino también, y especialmente, en otros ámbitos, como la educación, la formación, la salud y el trabajo social. De este modo, con la AS, la agricultura amplía su ámbito de influencia, acercándose a un número creciente de población y haciendo cambiar su papel en la sociedad, ofreciendo al campesinado la oportunidad de modificar y diversificar su actividad e implementar la idea de Responsabilidad Social Empresarial (RSE) y de trabajo cooperativo en la explotación agraria.

Así pues, la AS puede ser considerada un proceso dinámico de interacción entre el campesinado, el mundo empresarial, la institución y la sociedad civil, cuya participación varía, como veremos más adelanta, en función de las estructuras de cada país, a fin de plantear estrategias alternativas de desarrollo económico y social ene 1 seno de las comunidades locales a través de la innovación social y empresarial.

\section{LA DIMENSIÓN DE LA AGRICULTURA SOCIAL EN EL CONTEXTO EUROPEO}

Aunque podemos decir que es un fenómeno muy dinámico en Europa, se hace difícil cuantificar su práctica, ya que la falta de una categorización y de una codificación clara y estandarizada sobre qué es y qué no es Agricultura Social, hace que muchas iniciativas pasen desapercibidas y se contabilicen de diferente manera (o no se contabilicen) en función del país.

La caracterización de los proyectos de AS en el contexto europeo muestra una importante complejidad debido a su gran heterogeneidad. Esta situación dificulta la posibilidad de presentar estadísticas fiables y que respondan de una manera veraz a la realidad de esta actividad en el conjunto de Europa. Sin embargo, en el marco del proyecto Sofar ${ }^{1}$ se muestran algunos datos orientativos sobre la dimensión del AS en los diferentes países que participaban en el proyecto (Di Iacovo, O'Connor, 2009). Se trata de datos aproximados y no actualizados, por la dificultad de censar todas las iniciativas, pero que permiten captar la magnitud del fenómeno en un reducido grupo de países pero en los cuáles esta práctica está implantada en mayor grado (Cuadro 1). Hay que decir que el impulso que ha experimentado la AS en los último años modificaría sustancialmente estas cifras, aumentando el número de iniciativas y presencia

1 Sofar, Social Servicies in Multifunctional Farms ('SocialFarming') es un proyecto europeo que se realizó entre lso años 2005 y 2008 y que tenía como objetivo construir un entorno institucional para la Agricultura Social en Europa, impulsando la creación de vínculos entre agentes de diferentes países, acercando las diversas experiencias existentes para comprar, intercambiar y coordinar acciones en el contexto comunitario. Para más información se puede consultar la página web del proyecto y descarga los materiales resultantes: http://sofar.unipi.it/ 
en los territorios donde ya era presente y extendiéndose de manera significativa a otros países en los que aún no había indicios de tal actividad.

Las diferencias en los datos presentados no sólo son causadas por una variación en la implantación del AS, sino que se explica por lo comentado anteriormente: la dificultad de establecer un criterio único para su clasificación, haciendo que cada país incluya dentro de la etiqueta AS iniciativas de tipología y características diferentes. Sin embargo, a final del pasado año, y frente al interés creciente en diferentes países de la Unión Europea, el Comité Económico y Social Europeo aprobó un dictamen sobre la AS, a partir del cual se pretende, entre otras recomendaciones, reconocer la AS como un sector estratégico y crear un marco reglamentario que lo regule, unir esfuerzos desde las administraciones de diferentes países miembros, crear redes y fomentar la cooperación para su impulso, crear una base de datos, unificando estadísticas y criterios en la recogida de los datos, y promover la investigación en torno a la agricultura multifuncional y los servicios asistenciales. En definitiva, crear un entorno propicio para el impulso y el desarrollo de la AS en Europa ${ }^{2}$.

Cuadro 1. Aproximación a la dimensión y tipología de las iniciativas de AS en Europa

\begin{tabular}{|l|c|c|c|c|}
\hline \multicolumn{1}{|c|}{ PAÍ́ } & PÚBLICA & PRIVADA & OTRAS & TOTAL \\
\hline Bélgica & 258 & 38 & 12 & 308 \\
\hline Francia & 200 & 0 & 700 & 900 \\
\hline Alemania & 12 & 150 & 0 & 162 \\
\hline Irlanda & 2 & 92 & 12 & 106 \\
\hline Italia & 150 & 75 & 450 & 685 \\
\hline Países Bajos & 746 & 83 & 10 & 839 \\
\hline Eslovenia & 4 & 6 & 5 & 15 \\
\hline TOTAL & $\mathbf{1 3 7 2}$ & $\mathbf{4 4 4}$ & $\mathbf{1 1 8 9}$ & $\mathbf{3 0 1 5}$ \\
\hline
\end{tabular}

Fuente: Proyecto Sofar (Di Iacovo, O’Connor, 2009).

A continuación, y con la voluntad de captar esta heterogeneidad, describiremos las particularidades de las experiencia de AS en algunos países europeos como estas presentan substanciales diferencias en función del contexto en que fueros creadas (Di Iacovo, O'Connor, 2009).

2 Dictamen de Comité Económico y Social Europeo sobre el tema "La agricultura social: los servicios asistenciales ecológicos y las políticas sociosanitarias" (Dictamen de iniciativa). Bruselas, 12 de diciembre de 2012 (DOUE, 2013/C 44/07, 15.02.2013, p. 44-48) 
La mayor parte de iniciativas de AS en los países Bajos y Bélgica, donde este tipo de proyectos tienen una elevada presencia, son de carácter privado; explotaciones familiares gestionadas por personas con perfile profesionales y formación muy diversa. El carácter familiar de las iniciativas hace que la actividad principal sea la producción agrícola, y que la atención sociosanitaria y los servicios sociales aparezcan como una práctica secundaria y complementaria a la actividad agraria.

En el caso de Irlanda y Eslovenia, las iniciativas AS se inscriben principalmente en el Tercer Sector Social, las cuáles son desarrolladas por grupos religiosos, asociaciones creadas por familiares o las propias personas usuarias o por entidades sin ánimo de lucro. Normalmente cuentan con el apoyo de las instituciones públicas debido a la socialización de servicios de atención, haciendo que en la mayoría de los casos la producción agrícola se convierta en la actividad secundaria y realmente sean los servicios sociales la actividad principal.

En Alemania, por el contrario, una legislación específica sobre los servicios y los requisitos para impulsar iniciativas con personas con discapacidades. Estas leyes rigen el salario, la jornada laboral y la dimensión de los centros donde estos colectivos trabajan como medida para evitar los abusos de las empresas convencionales. Sin embargo, esto dificulta la incorporación de personas con discapacidad en las explotaciones agrarias de carácter familiar motivado por las limitaciones legales.

En Italia los proyectos de AS con una larga tradición, remontándonos a la década de 1970 cuando surgen las primeras cooperativas agrarias con carácter social. Existen dos tipos predominantes de iniciativas de AS: por un lado, las que se centran en la atención y, por la otra, las que lo hacen en la integración laboral. Sin principalmente empresas, a menudo cooperativas, sin ánimo de lucro y que están fuertemente arraigadas en el tejido asociativo local, vinculadas en redes de dinamización agroecológica y de consumidores que esperan un producto con responsabilidad social.

Las experiencias de AS en Francia se integran en diversas redes de carácter estatal y regional que tienen como base el trabajo de personas desfavorecidas o con problemáticas específicas a huertos y ajardines comunitarios que emplean la agricultura como herramienta de inserción sociolaboral.

Por último, en el caso de España se ha detectado la existencia de un grupo reducido de experiencias de AS aisladas, distribuidas por el territorio y con escasas conexiones entre ellas debido de la inexistencia de redes. En Cataluña, en cambio, el impulso que ha experimentado el AS ha hecho surgir un importante número de iniciativas, las cuales se empiezan a organizar, tejiendo vínculos y estableciendo sinergias a través de la creación de redes y programas específicos. En este sentido, se puede destacar la Xarca AgroSocial (Red Agrosocial), impulsa por la fundación de una entidad de crédito, la cual tienen como 
objetivo incentivar el desarrollo de esta actividad en el territorio catalán y promover la cooperación entre las diferentes experiencias que son miembros, que actualmente supera la treintena. Ante este auge del emprendimiento social y de la revalorización de la agricultura como sector económico estratégico, algunos órganos de gobierno supralocal, como la Diputación de Barcelona, han apostado por la formación de técnicos de ayuntamientos y consejos comarcales con el fin que estos fomenten la inclusión de proyectos de AS en los instrumentos de desarrollo local de sus respectivos municipios.

\section{LA AGRICULTURA SOCIAL: DESARROLLO LCOAL Y DINAMIZACIÓN AGROECOLÓGICA}

En las aportaciones realizadas por diversos autores se muestra un estrecha relación entre el desarrollo local y AS (Di Iacovo, O'Connor, 2009; Di Iacovo, 2010; Dessin, Bock, 2010). Los proyectos de AS representa un fuerte impulso para el desarrollo económico y social a nivel local, principalmente en áreas rurales, donde las actividades ligadas al territorio y vinculadas a productos de la tierra tienen una mayor repercusión en el desarrollo económico endógeno, la cohesión social y la consolidación del tejido empresarial. Se tratan de iniciativas socialmente más justas, pero también más respetuosas con el medio ambiente y el territorio, fomentando una serie de valores que contribuyen positivamente a la construcción de nuevos modelos de desarrollo. Revalorizan la agricultura y promueven su multifuncionalidad, al mismo tiempo que ponen en valor los recursos del propio territorio en que se instalan, generando a través de ellos actividad económica productiva e impulsando mejoras sociales. De esta forma, podemos decir que las iniciativas de AS contribuyen a dinamizar el medio rural, fomentando la fijación de población, la generación de nuevos yacimientos de empleo y la construcción de una sociedad más cohesionada e igualitaria.

Buena parte de las iniciativas, por el talante de sus impulsores, apuestan por una agricultura ecológica, orgánica o biodinámica, o en todo caso, por un modo de producción integrado o convencional, en el cuál se intenta minimizar al máximo el uso de productos químicos, siendo el respeto por el entorno uno de los pilares en los que se sustentan. Asimismo, la sensibilidad de las personas que impulsan o dirigen explotaciones vinculadas con la AS hace que estas iniciativas sean un exponente, no sólo en el desarrollo social y económico del lugar, sino también en la revalorización de la cultura y las costumbres locales. Perciben el legado cultural local como un activo, que no sólo les proporciona singularidad y productos únicos de cara a un mercado cada vez más exigente, sino que les permite adaptarse al entorno y planificar la explotación en función de las características locales y los ciclos naturales. En este sentido, algunos de estos proyectos han realizado una formidable, tarea, por ejemplo, en la recuperación de variedades locales, de técnicas tradicionales de cultivo y de 
recuperación de campos abandonados; prácticas que se habían ido perdiendo progresivamente y que tradicionalmente han sido una eficaz herramienta en la conservación del territorio y el paisaje. De esta forma se recuperan funciones y dimensiones que antaño tenía la agricultura y que se habían ido desdibujando desde mediados del siglo XX con la implantación de parámetros y métodos de cultivo dictados por la agricultura industrial.

Por otra parte, y como ya hemos comentado anteriormente, los proyectos de AS son un exponente del surgimiento de un nuevo campesinado, el cual opta por una producción loca, que permita conectarse directamente con los consumidores, con la intención de reconfigurar el sistema agroalimentario actual, trabajando para alcanzar la soberanía alimentaria, a través de un consumo de proximidad de productos éticos, limpios y justos socialmente (Carbone, Senni, 2010).

Paralelamente a la emergencia de este nuevo paradigma campesino, que aporta "otra manera" de producir alimentos, aparecen nuevas pautas de consumo globales, las cuáles son, actualmente, especialmente visibles en los países postindustriales. Las sociedades de estos países están cada vez más sensibilizadas con el consumo de alimentos de calidad, saludables y que respeten el medio ambiente (Verbeke, 2005). Los hábitos de consumo de una parte de la sociedad muestran un interés creciente por la compra de alimentos ecológicos, éticos, saludables y de calidad. En España, el consumo de productos procedentes de la agricultura ecológica ha aumentado de manera significativa en los últimos años, situándose actualmente entorno al 2\%, y con un aumento en 2011 del $7 \%$ respecto al año anterior ${ }^{3}$. Aunque es un porcentaje reducido, sobre todo si tenemos en cuenta que España es el país con más superficie agraria dedica a producción ecológica, se percibe un crecimiento constante y con largo recorrido.

Los productos procedentes de las explotaciones que trabajan en la AS encajan perfectamente con estos hábitos de consumo, cubriendo las existencias de estos segmentos de mercado, emergentes pero cada vez más numerosos. Estas pautas de consumo ofrecen clara salida comercial a la producción de estos proyectos, promocionándoles posibilidades de negocio y su viabilidad económica. En la mayoría de los casos, la comercialización y distribución de los productos suele ser de proximidad, reduciendo al máximo los intermediarios existentes entre la explotación y el consumidor final. Los productos se venden directamente en la misma explotación, a través de cooperativas o grupos de consumo, en pequeños comercios y establecimientos especializados, acortando el ciclo comercial y garantizando la total trazabilidad del producto, lo que le da aún más valor añadido a la producción.

3 Datos sobre Agricultura Ecológica del Ministerio de Agricultura, Alimentación y Medio Ambiente (2011). 


\section{L'OLIVERA: VINO Y ACEITE ECOLÓGICOS CON VALOR SOCIAL}

L'Olivera es un proyecto de As constituido como cooperativa de integración social, que se dedica a la producción ecológica de vino y aceite en Vallbona de las Monges, un pequeño pueblo de la comarca del Urgell (Lleida, Cataluña), y que incorpora a personas con discapacidad psíquica que participan de manera activa en todo el proceso productivo (Figura 1).
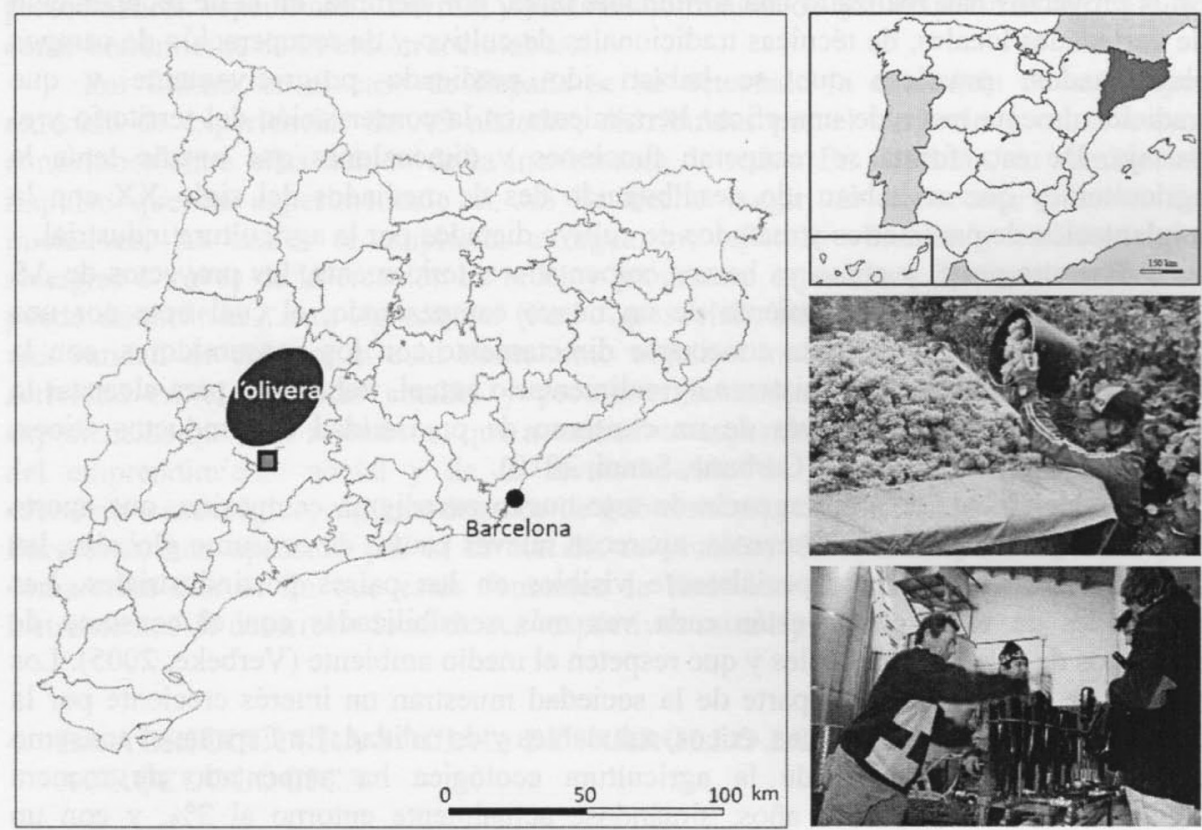

Figura 1. Localización e imágenes de la explotación y la bodega.

Fuente: elaboración propia y www.olivera.org

Se trata de una iniciativa que empezó su andadura a mediados de la década de 1970, cuando un grupo de jóvenes de origen urbano decidieron migrar hacia un territorio en pleno éxodo rural y una profunda crisis estructural, motivados por la posibilidad de construir algún modelo de vida alternativo estrechamente vinculado a la tierra, que se estructuraba entorno a dos pilares: la producción agraria y la vida en comunidad. Llegaron en un momento en que la población autóctona abandonaba campos de cultivo y cerraba casas para irse a la ciudad en busca de una nueva vida. A esta motivación se añade un importante componente de acción social, y es que uno de los objetivos era realizar el proyecto de manera conjunta con personas discapacitadas, esto es, en palabras de su director Carles Ahumada "no trabajando para ellas, son trabajando con ellas; 
considerando que todo el mundo tiene sus capacidades y sus discapacidades, y que en este sentido no se tenía por qué hacer ninguna diferencia"4.

Ya en sus inicios, l'Olivera tenía una clara voluntad de dinamización, no sólo agroecológica, sino también social y económica, del lugar en que se encontraba, que como muchos otros territorios rurales de la Península estaba en una situación desfavorable. La intención de crear una iniciativa para promover el desarrollo local endógeno llevó a sus impulsos a pensar posibles alternativas, concluyendo que era el sector agrario el recurso territorial que les acercaba a aquellos valores que perseguían y a partir del cual querían forjar su apuesta de desarrollo local. Durante la primera etapa estuvieron probado diferentes actividades, hasta que en 1985 deciden crear la bodega y empezar el proceso de producción vitivinícola. A partir de ese momento el proyecto coge forma y un año más tarde se constituyen como Centro Especial de Empleo (CEE), forma jurídica que recoge la LISMI $^{5}$ para favorecer la inserción sociolaboral de personas con discapacidad en España.

A principio de la década de 1990, la producción de vino alcanzaba ya las 14.000 botellas anuales, volumen que ha ido en aumento hasta la actualidad, situándose en torno a las 100.000 Botellas. Paralelamente se ha ido introduciendo el cultivo de olivos -recuperando campos en proceso de abandono- y la producción de aceite ecológico, hasta el punto que en 2005 deciden construir un molino propio para prensar las aceitunas en la misma explotación. El incremento de la producción les ha obligado a ampliar progresivamente las instalaciones, lo cual ha revertido positivamente en una mayor presencia comercial, aumentando el volumen de ventas y las cuotas de exportación. La buena marcha del proyecto, pese a las dificultades provocadas por la crisis, les ha permitido crear nuevos puesto de empleo y aumentar la dotación de servicios de atención para las personas con discapacidad de este territorio rural, como es la creación de un hogar-residencia y un centro de terapia ocupacional.

Actualmente la cooperativa cuenta con más de 45 trabajadores, de los cuáles, casi el $50 \%$ son personas con algún tipo de discapacidad psíquica y/o trastorno mental. La apuesta firme por la economía social y colaborativa ha dado lugar a un modelo organizativo que integra de manera plena a todos los trabajadores, sin excepción de condiciones; dando a las personas con discapacidad la posibilidad de ser socios de la cooperativa, con plenos derechos si así lo desean. Este hecho genera confianza e implicación en el proyecto, aportando

4 En el proyecto que llevamos a cabo desde el Departament de Geografia de la UAB y la Fundació CEDRICAT, Y QUE LLEVA POR TÍTULO "La Agricultura Social en el desarrollo local y en la ocupación de colectivos en riesgos de marginación social", hemos recogido el testimonio de diferentes iniciativas de AS en Cataluña a través de entrevistas en profundidad y cuestionarios. Una de ellas fue l'Olivera Cooperativa, y es por eso que adjuntaremos algunos fragmentos de la entrevista mantenida con su directos Carles Ahumada para apoyar algunas de nuestras explicaciones.

5 Ley para la Integración Social de los Minusválidos (Ley 13/1982, de 7 de abril). 
a los colectivos que ocupa una experiencia laboral y de integración social real, en la que a través del trabajo que realizan se sientes valorados profesionalmente, integrados a la comunidad y personalmente capaces de llevar una vida normalizada.

La tarea desempleada por l'Olivera en materia de desarrollo local, y retomando el carácter multifuncional de la agricultura, y en concreto de los proyectos de AS, no acaba en la producción de vino y aceite y la integración sociolaboral de personas con dificultades, sino que también ha generado nuevas actividades en el ámbito de la economía social, estableciendo vínculos con otras iniciativas económicas con valor social y creando redes de comercialización conjuntas. Estos proyectos se complementan con el turismo, ofertando actividades enoturísticas y visitas a la bodega que atraen visitantes a la comarca, combinando su estancia con la visita al conocido monasterio cisterciense de Vallbona de les Monges, situado en la misma localidad.

En este sentido, l'Olivera aparece como uno de los referentes de la AS en Cataluña, incorporando a personas en riesgo de marginación social a un proyecto real de desarrollo rural -económicamente viable, socialmente justo y ambientalmente sostenible-; demostrando que es posible crear estrategias de desarrollo local que combinen economía social y dinamización agroecológica. Además, la simbiosis entre acción social y agricultura social y dinamización agroecológica. Además, la simbiosis entre acción social y agricultura está experimentando un importante aumento en Cataluña, mostrando como en los últimos años, especialmente desde el inicio de la crisis económica, han aparecido un buen número de proyectos de AS de nueva creación.

\section{A MODO DE CONCLUSIÓN}

Las iniciativas de AS tienen un papel crucial en la recuperación de la agricultura en los países occidentales, una recuperación física, de campos de cultivo y fincas agrícolas, y simbólica, por su contribución a la revalorización de la vida de campesino y por la labor de sensibilización de la importancia estratégica que tienen este sector en el desarrollo económico y social de cualquier territorio. Muchos de los proyectos de AS han aparecido en territorios donde la agricultura, y el sector primario en general, hacía tiempo que era un sector económico en clara recesión. En otros lugares, donde la base económica y a proporcionar nuevos modelos de gestión de las explotaciones agrarias, demostrando la multifuncionalidad de la agricultura y ofreciendo productos con valor añadido, tanto económico como socialmente.

El desarrollo local en el mundo rural ha estado estrechamente vinculado al turismo en las últimas décadas, y aunque en los últimos años ya se han apuntado cambios de tendencia. Muchas de las estrategias siguen basándose en la atracción de visitantes a través de valores relaciones con la tradición, la 
autenticidad y la singularidad de estos espacios (Barrachina, Guirado, Tulla, 2010). La crisis económica y financiera, pero también social y de valores, que viven principalmente los países del sur de Europa ha hecho visibles estas debilidades del modelo de desarrollo que hasta hoy ha imperado en la mayoría de áreas rurales. En este sentido, nos encontramos en un momento óptimo para trazar rutas hacia modelos de desarrollo mucho más ligados al territorio, que constituyan a la economía del bien común, y que sean menos dependientes de los ciclos económicos y de las burbujas especulativas.

Es obvio que la AS se presenta como una clara muestra de innovación económica y social en el ámbito el desarrollo local, ya que está estrechamente relacionada con la acción colectiva, condicionada por la estructura social de los territorios en los que se desarrolló, y con la interacción activa, participativa y permanente entre personas, agentes y recursos territoriales, dando lugar a un mundo rural vivo, dinámico y socialmente cohesionado. El hecho que la agricultura $-\mathrm{y}$ por extensión a la actividad agraria- esté vinculada necesariamente con el territorio hace que su práctica contribuya a tejer redes de colaboración y cooperación a nivel local que promueven la cohesión social y ayudan a fijar población en el territorio, haciendo crecer el sentimiento de pertenencia a una comunidad y fomentando el trabajo cooperativo. En este sentido, la AS aparece como un elemento de organización social, de valorización y de (auto) gestión de los recursos locales, que pretende ser una alternativa a las estructuras agrarias convencionales y los modelos de desarrollo local imperantes, así como una oportunidad de dotación de servicios dirigidos a la atención y ocupación de personas en riesgo de marginación social del mundo rural.

\section{BIBLIOGRAFÍA}

ARMESTO, X. (2005). "Notas teóricas en torno al concepto de postproductivismo agrario". Investigaciones Geográficas, 36: 137-156.

BARRACHINA, M.: GUIRADO, C.; TULLA, A.F. (2009). "Viejos problemas, nuevas respuesta: las áreas De montaña frente a las debilidades del sistema neoliberal. El caso del Pirineo Catalán". PILLENT, f., CAÑIZARES, M. C.; RUIZ, A. R. (coord.). Geografía, territorio y paisaje: el estado de la cuestión: Actas del XXI Congreso de Geógrafos Españoles. Ciudad Real, 27-29 de octubre de 2009 (p. 85-97).

BOWLER, I.R. (1996). "Agricultural land use and the post-productivist transition" LÓPEZ ONTIVEROS, A.; MOLINERO, F. (eds.). La investigación hispano-británica reciente en Geografía Rural: del campo tradicional a la transición postproductivista. Murcia: Asociación de Geógrafos Españoles, p. 179-187.

CARBONE, A.; GAITO, M.; SENNI, S. (2007). "coNSUMERS" Buying in the Short Food Chains: Aternatives for Trust". 1st Internacional European 
Forum on Innovation and System Dynamics in Food Networks. InnsbruckIgls, Austria: February 15-17, 2007.

CARBONE, A.: SENNI, S. (2010), "Consumers" Attitude Towars Ethical Food: Evidence from Social Farming in Italy". DESSIN, J.; BOCK, B.B. (eds.). The Economics of Green Care in Agriculture. Loughborough University, p. 76-77.

COLLANTES, F. (2005). "Declive demográfico y cambio económico en las áreas de montaña españolas, 1860-200". Revista de Historia económica, 23 (3): 515-540.

DESSIN, J., BOCK, B. (2010) (eds.). The Economics of Green Care in Agriculture. Loughborough: Loughborough University.

DI IACOVO, F. (2010). "l'agricoltura sociale: patriche e paradigmi nello scenario comunitario". XLVII Convegno di Studi de la Società Italiana di Economia Agraria "L'agricoltura oltre le crisi" 22-25 settembre de 2010. Campobasso: Università degli Studi del Molise.

DI IACOVO, F.; O'CONNOR, (eds.) (2009). Supporting Pilicies for Social Farming in Europe. Progressing Multifunctionality in Responsive Rural Areas. FIrenze; ARISA.

LOCKWOOD, J. A. (1999). "Agricultura and biodiversity: finding our place in this world". Agriculture and Human Values, 16: 365-379.

LÓPEZ, J. A.; FERNÁNDEZ, D. (2003). Con la comida nos e juega: Alternativas autogestionarias a la globalización capitalista desde la agroecología y el consumo. Traficantes de Sueños. Madrid.

MEDINA, F. x. (2010) "introducció: reflexions sobre les alimentacions contemporànies. MEDINA, F. X. (ed.) Reflexions sobre les alimentacionscontemporànies. De les biotecnologies als productes ecològics. Barcelona: Editorial UOC.

MONLLOR, N. (2011). Explorant la jove pogesia: camins, practiques i actituds en el marc d'un nou paraigma agrosocial. Estudi comparatiu entre el sud-oest de la provincia d'Ontario y les comarques gironines. Teis doctoral. Unviersitat de Girona.

O’CONNOR, D.; LAI, M.: WATSON, S. (2010). Overwiev of Social Farming and Rural Development Policy in Selected EU Member Sates. NRN Joint Thematic Initiative on Social Farming. European Network for Rural Development.

PLOEG, J. (2010). Nuevos campesinos: campesinos e imperios alimentarios. Editorial Icària.: Barcelona.

PLOEG, J. D. (2006). "Agricultural production in crisis". CLOKE, P.; MARSDEN, T.; MOONEY, P. (eds.). Gandbook of Rural Studies. SAGE Publications: London, p. 258-277.

SEVILLA, E. (2007). De la socieología agraria a la agroecología. Icària Editorial: Barcelona. 
VERBEKE, W. (2005). "Agriculture and the food industry in the indormacion age". European Review of Agricultural Economics, vol 32(3): 347-368.

WOODS, M. (2005). Rural Geography: processes, responses and esperiences in rural restructuring. SAGE Publications: Londres.

\section{Agradecimientos}

Esta comunicación se elabora en el marco del proyecto La Agricultura Social en el desarrollo local y en la ocupación de colectivos en riesgo de marginación social, financiado por el Programa RecerCaiza (2011ACUp00023) y realizado conjuntamente por el Departament de Geografia de la UAB y la Fundació CEDRICAT. El equipo del proyecto lo forman A. Badia, M. Barrachina, C. Guirado, A. F. Tulla (IP), N. Valldeperas y A. Vera (DEpartament de Geografia, UAB), y C. Evard, I Pallarès y M. Rancaño (CEDRICAT). 